\title{
ZVS \& ZCS Based Converter for Efficiency Regulation
}

\author{
T. Bharani Prakash ${ }^{1}$, R.Pari ${ }^{2}$, M.Baby ${ }^{3}$ \\ ${ }^{1,2}$ Assistant Professor, Department of Electrical and Electronics Engineering, V.S.B Engineering College, \\ Karur-639111, Tamilnadu, India \\ ${ }^{3}$ P.G Scholar, Power Systems Engineering, V.S.B College of Engineering, Karur -639111, Tamilnadu, India
}

\begin{abstract}
- a new high-efficiency regulation method for a zero-current and zero-voltage current-fed pushpull converter is presented. The method proposed is based on the use of a controlled transformer as a postregulator, which adds or subtracts an additional voltage to the output filter of the converter. An auxiliary regulator, which only handles a percentage of the output power, controls this transformer, and therefore, results in much higher efficiency than a normal preregulator would have. The novelty of the presented converter is that regulation is achieved without affecting the zero-voltage and zero-current switching in any working conditions. Small- and large-signal models of the converter have been developed and a low-power prototype has been simulated and implemented. The good agreement, between the experimental simulations and theoretical results, validates the theoretical developments.
\end{abstract}

Keywords-DC-DC power conversion, parallel regulation, resonant power conversion, travelling-wave amplifiers, zero-voltage zero-current switching (ZVZCS).

\section{INDRODUCTION}

ISOLATED high-voltage dc/dc converters are widely used in high-voltage dc/dc converters are widely used in travelling-wave tubes, electrical propulsion, ozone generation, medical X-ray imaging, lasers, etc. In medium-power applications, current-fed push-pull [1]-[3] or current-fed half-bridge [4], [5] are the most common topologies, whereas current-fed full-bridge topology is the most frequently used for high-power applications [5]-[9]. The main advantages of these topologies are high-voltage conversion ratio, continuous input current, galvanic isolation between input and load, and no need of an output filter inductor.

In order to increase the power density and minimize the size and weight of these converters, the general practice, is to increase their operation frequency; however, the higher switching frequency, combined with the effects of the parasitic elements of the converter, the larger the switching losses and electromagnetic interferences (EMI). Therefore, many soft-switching techniques have been proposed in the literature to reduce switching losses and EMI, the most popular ones are zero-voltage switching (ZVS), zero-current switching (ZCS), and active clamp techniques [1]-[9].

A different approach of a current-fed push-pull resonant converter was proposed in [9]. It is based on the use of the "zero-voltage and zero-current power switching (ZVZCPS)" technique. This new converter was developed to decrease the mass of the high-output voltage dc/dc converters used for the power supply of the travelling-wave tube amplifier (TWTA) in aerospace applications. The main advantages of this converter, compared to conventional current-fed push-pull converters and the ones with active-clamp circuit presented in [2] and [3] is that the main switches always operate under both ZVS and ZCS when switched ON and switched OFF. This advantage allows achieving smaller and more efficient converters.

\section{Z ZVS \& ZCS TECHNIQUE}

The advantages of the ZVZCPS push-pull are listed as following.

1) All the parasitic capacitances and inductances are used in a resonant fashion.

2) Main switches operated under both ZVS and ZCS when switched ON and switched OFF.

3) Output rectifier operated under ZCS.

4) Very small number of components.

5) Independence of ZVS and ZCS respect to load changes.

6) Very high efficiency (better than 98\%).

7) Very simple low loss gate driver.

8) Operation at fixed frequency.

9) Very low EMI noise generation. 
The major drawback of this topology is that it deals in a non-regulating converter, and its output voltage is only determined by the input voltage and its main transformer turns ratio. To achieve a regulated output, coping with changes in the input voltage, it is necessary to use an additional converter in series with the ZVZCPS [1]. Usually a buck converter is used as a preregulator to control the voltage at the input of the resonant stage. The addition of a buck converter in series with the ZVZCPS is known, by the European Space Agency, as the standard multiple application regulator (SMART) converters. Nowadays, this topology is widely used in space applications to supply the TWTAs and the ion-thrusters power supplies. In Figure 1, it is presented the basic schematic of the SMART converter. Obviously, the addition of a buck converter produces an efficiency penalty, around $10 \%$ for nonregulated inputs.

To improve the efficiency of the SMART converter two techniques have been proposed in [7]. In both of them, the series regulator is removed and a parallel power processing technique is used to regulate the output. With the parallel power-processing scheme, the regulation is achieved only by controlling a percentage of the total output power, and hence, the power regulator scores very high efficiency while retains output regulation capability [10]. A similar concept has been recently applied to the LLC resonant converter with the name of quasi-parallel converter and Sigma dc/dc conversion.

In [6] and [8], the regulation is achieved by adjusting the input voltage of the dc/dc transformer of the LLC converter with a low-power converter. In [9], the parallel power-processing principle is based on the use of the transformer turns ratio regulator \{TR 2 concept [7]. The principle employed in the \{TR 2 is based on the addition or subtraction of an ac voltage in the ac path of the converter. In [5], the \{TR 2 technique is applied in the primary side of the push-pull regulator. With this technique, the efficiency is improved, but the resonance of the ZVZCPS is affected by the introduction of the \{TR 2 transformer, losing the ZVS when the input voltage is different from the nominal one used for the design of the ZVZCPS. In [8], the principle employed is similar to the previous one, but instead of using the $\{\mathrm{TR}\} 2$ technique, it uses a normal transformer to add an ac voltage in the primary ac path of the push-pull regulator. In this way, the efficiency is improved, but the resonance is affected, losing again the ZVS.

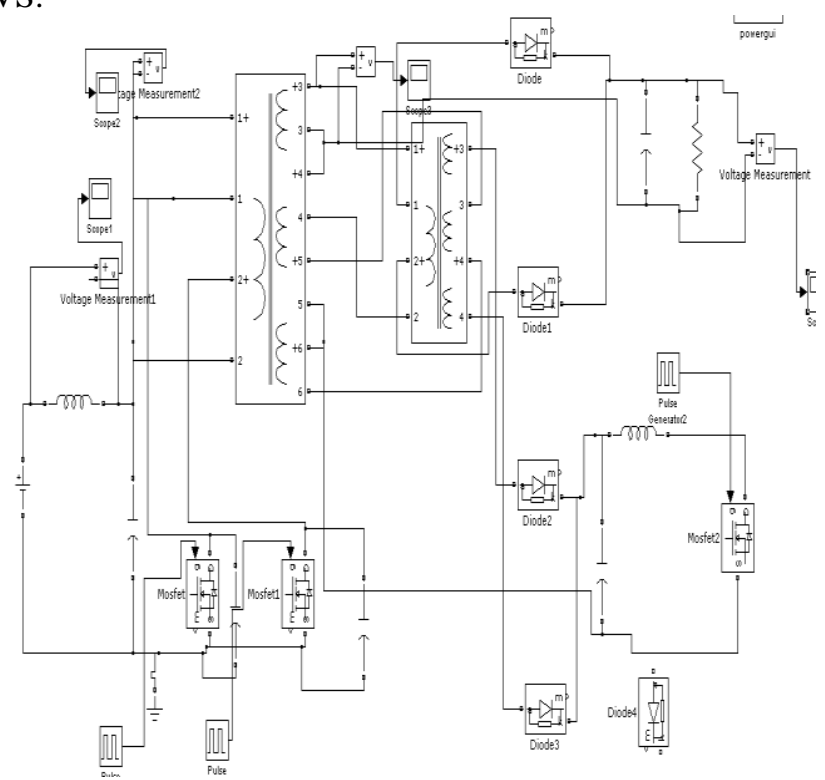

Figure 1.Open loop circuit

This paper proposes a different way to apply the parallel power-processing technique to the ZVZCPS converter. The method proposed is based on the use of a controlled transformer as a post regulator, adding or subtracting an additional voltage to the output filter of the converter. 


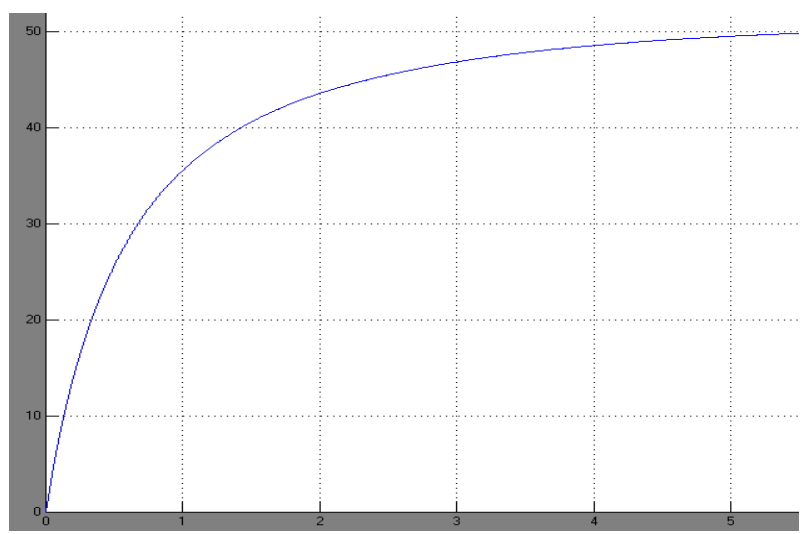

Figure 2.Open loop Waveform

This technique has already been applied to a classical forward converter with two outputs, but the novelty of this paper is the implementation of such technique to the ZVZCPS converter, demonstrating than the zero-voltage and zero-current switching is maintained under any operating conditions and improving the efficiency when compared against the SMART converter in the whole input voltage range. In Section II, the operation of the proposed converter is presented. In Section III, the small-signal analysis of the converter is derived and in Section IV, the simulation and experimental results of a $250 \mathrm{~W}$ prototype are shown. The paper is summarized in Section V and ends with the conclusions.

\section{PROPOSED CONVERTER}

The proposed converter uses the $\{\mathrm{TR}\} 2$ technique to add or subtract an ac-controlled voltage to the output filter. In Figure 2, it is presented the technique of the $\{T R\} 2$ regulation method applied in the secondary side of an isolated dc/dc converter. In Figure 2, only the ac part of the converter is shown. In Figure 2, VC is the rms value of the controlled ac voltage obtained from a transformer turns ratio limit the voltage regulation. If VC varies between zero and VS.

This allows a regulation of the output auxiliary PWM-controlled converter. VS is the rms value of the ac voltage obtained from the non-regulated input and $\mathrm{VO}$ is the rms value of the regulated ac output. The main transformer of the converter has a turns-ratio relationship, from input to output, equal to 1: NO. To apply the $\{T R\} 2$ regulation, an additional winding is needed in the main transformer, with a turns ratio 1: NM, regarding to the primary side. To add or subtract the controlled voltage, a small regulating current transformer with a turns ratio 1: NR is devised.

Now, it is possible to regulate the output voltage by changing the control voltage VC and this is achieved varying the duty cycle of the auxiliary converter. Nevertheless, the input voltage and the against a $20 \%$ change at the input voltage, while only handling, in the auxiliary converter, the $20 \%$ of the output power. This proportion of power $(\mathrm{k})$ is added or subtracted with the efficiency of the auxiliary controller $(\eta \mathrm{R})$ so the overall efficiency of the converter is given by For instance, if a regulator handles $20 \%$ of the power with an efficiency of $90 \%$, the regulation only decreases the overall efficiency of the original converter by $2 \%$. This strategy results in much higher efficiencies than the two-stage systems [1], where the efficiency penalty of introducing a $90 \%$ efficiency series regulator is $10 \%$. Further, the addition of the regulating transformer in the secondary side of the ZVZVPS does not affect the resonant operation of the converter for any input voltage, as presented in the following. The implementation of the proposed converter is shown in Figure 3. The converter consists of a conventional ZVZCPS, an auxiliary boost converter to obtain the control voltage, an additional winding in the main transformer and a regulating transformer. Figure 4 illustrates the equivalent circuits for each operating intervals and it depicts the main waveforms of the converter.

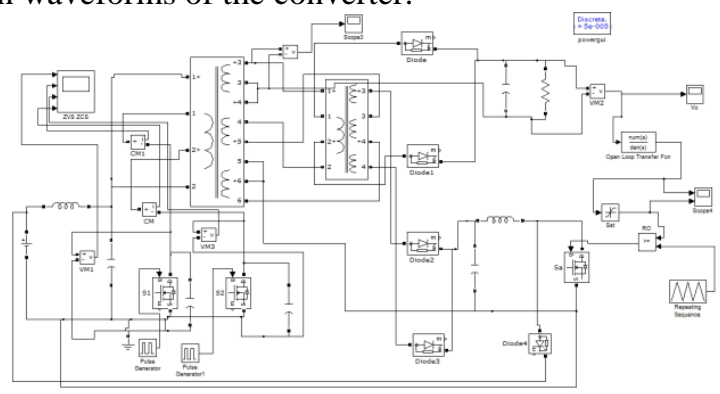

Figure 3.Closed loop 
Although in [9], a detailed mathematical analysis of the ZVZCPS waveforms can be found, they are briefly described as following.

Interval 1 [t0 - t1]: This interval starts by turning the switch a M1 ON when its voltage is zero. The current through M1 increases in a resonant fashion because Ctun resonates with the leakage inductance of the main transformer, producing a half sinusoidal current through M1, D1, and D3. The parasitic capacitance of M2 (Cds2) is charged to twice the Ctun voltage. The magnetizing inductance (Lm) of the main transformer is charged and the $\{\mathrm{TR}\} 2$ regulation is applied through D3 and the regulating transformer.

Interval 2 [t1- t2]: When the current through M1, D1, and D3 falls to zero, the switch M1 is turned OFF. In this interval, the energy stored in Lm forces to discharge the parasitic capacitance on M2 (Cds2), charging the parasitic capacitance of M1 (Cds1) with a constant current. During this interval, there is no transfer of energy from the input to the output and all the diodes are OFF. Ctun is charged with the input current of the converter Iin.

Interval 3 [t2 - t3]: When the voltage through M2 falls to zero, the switch M2 is turned ON. The current through M2 increases in a resonant fashion because Ctun resonates with the leakage inductance of the main transformer, producing a half-sinusoidal current through M2, D2, and D4. The parasitic capacitance of M1 (Cds1) is charged to twice the Ctun voltage. The magnetizing inductance (Lm) of the main transformer is discharged and the $\{T R\} 2$ regulation is applied through D4 and the regulating transformer.

Interval 4 [t3 - t4]: When the current through M2, D2, and D4 falls to zero, the switch M2 is turned OFF. Within this interval, since both switches are OFF, the energy stored in Lm forces to discharge the parasitic capacitance on M1 (Cds1), charging the parasitic capacitance of M2 (Cds2) with a constant current. During this interval, there is no transfer of energy from the input to the output and all the diodes are OFF. Ctun is charged with the input current of the converter Iin.

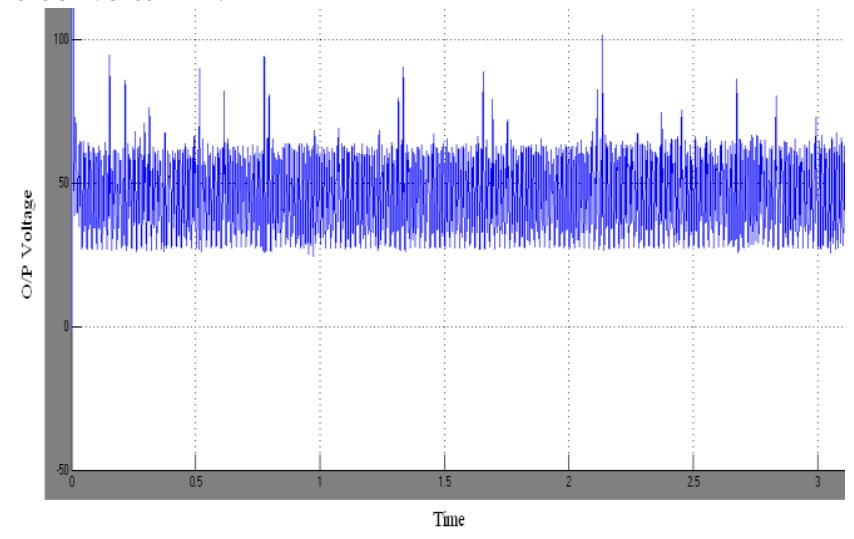

Figure 4.closed loop Waveform

As it can be seen, if the converter is properly tuned, working with a constant frequency and a constant duty cycle in the main switches, all the switches work in a resonant fashion. If VS changes, the resonance is not affected. This is because during the ON time VS does not influence in the resonance, and during the gap time, although the parasitic capacitances have been charged to twice the new VS.

The magnetizing current of the main transformer has changed too, and therefore, the gap time needed to charge/discharge the parasitic capacitance is not affected either. This is the main advantage of introducing the $\{T R\} 2$ regulation in the secondary side of the main converter, the ZVS and ZCS is not affected by the changes of the input voltage.

\section{SMALL-SIGNAL ANALYSIS}

The transfer function of the output voltage versus the auxiliary converter duty cycle is needed to design the control loop of the proposed converter. The transfer function is derived following the guidelines presented in [3]. Since the auxiliary transformer only adds or subtracts a voltage to the output voltage, it can be considered as a dc transformer. The dependence of the output voltage with the control voltage can be calculated from the dc and ac models of the converter. In this model, an input inductance parasitic resistance (RL) has been considered for taking into account the damping effects. 
From the ac and dc models, the transfer function can be derived as shown at the bottom of the next page. On the other hand, the auxiliary boost converter, see Figure 3, and has the following transfer function of the control voltage versus the auxiliary converter duty cycle in continuous conduction mode.

TABLE I PROTOTYPE SPECIFICATIONS

\begin{tabular}{|l|l|}
\hline \multicolumn{1}{|c|}{ Parameter } & \multicolumn{1}{c|}{ Value } \\
\hline Power & $50 \mathrm{~W}-250 \mathrm{~W}$ \\
\hline Vin & $50 \mathrm{~V} \pm 5 \mathrm{~V}$ \\
\hline Vout & $50 \mathrm{~V}$ \\
\hline Bus voltage ripple $\left(\Delta \mathrm{V}_{\mathrm{BUS}}\right)$ & $<1 \%$ Vout \\
\hline $\begin{array}{l}\text { ZCZVPS mosfets } \\
\text { switching frequency }\end{array}$ & $100 \mathrm{kHz}$ \\
\hline $\begin{array}{l}\text { Auxiliary boost converter } \\
\text { switching frequency }\end{array}$ & $200 \mathrm{kHz}$ \\
\hline
\end{tabular}

The final transfer function, needed for the control-loop design. As represented in Figure 3, to achieve the voltage regulation a PID controller is used, obtaining the following open-loop transfer function.

The open-loop transfer function of this converter is similar to the buck converter; it only differs in the extra resonant capacitor and the components LB and CB from the auxiliary converter. However, due to the function of these components, the double-pole and double-zero will appear at frequencies higher than the double-pole introduced by the main filter and it does not affect to the stability margins.

\section{SIMULATION AND EXPERIMENTAL RESULTS}

In order to verify the experimentally, the behavior of the proposed converter a $250 \mathrm{~W}$ prototype has been designed, simulated, and built. This converter has been designed to regulate the output of a sequential switching shunt maximum power point regulator (S3MPR) to $50 \mathrm{~V}$. The S3MPR output voltage always varies following the maximum power point (MPP) voltage of the solar array sections. The prototype has been designed to cope with an input from 45 to $55 \mathrm{~V}$.

(i.e.,) The expected variation of the MPP voltage of the spacecraft solar arrays under constant irradiance conditions during its lifetime. The switching frequency of the ZVZCPS push-pull has been fixed at $100 \mathrm{kHz}$ because it is the standard in the latest power converters for TWTAs and allows achieving simultaneously low mass and high efficiency.

The main specifications of the prototype are summarized in Table I. As it can be seen in the specifications, the auxiliary boost converter has to regulate the output against a $20 \%$ variation of the input voltage, while only handling the $20 \%$ of the output power.

The design of the resonant stage has been done. The main transformer has been implemented in an ETD49 core shape, using 3C90 material with eight turns in each winding $(\mathrm{NO}=1)$. The measured magnetizing inductance of the transformer was $307 \mu \mathrm{H}$ and the leakage inductances $1.1 \mu \mathrm{H}$. The transistors used are IRFP250 from International Rectifier with measured parasitic capacitance of $1.4 \mathrm{NF}$. Taking in mind these values, the rest of the converter has been designed $(\mathrm{TO} \mathrm{N}=4.2 \mu \mathrm{s}$, Tgap $=1.8 \mu \mathrm{s}, \mathrm{Ctun}=360 \mathrm{NF}$, Lin $=200 \mu \mathrm{H}$, and Cout $=150$ $\mu \mathrm{F})$.

The auxiliary boost converter is synchronized with the resonant stage. It works at twice the push-pull switching frequency. Special care has been taking in designing the capacitor and the inductor of the boost converter. The boost capacitor multiplied by the transformer ratio should be much higher than the tuning capacitor in order to avoid interference in the resonance behavior $(\mathrm{CB}=200 \mathrm{nF})$. The boost inductor has been designed to assure continuous conduction mode (CCM) when the output power is bigger than $50 \mathrm{~W}$ ( $\mathrm{LB}=200$ $\mu \mathrm{H}) . \mathrm{CCM}$ is not mandatory, but the open-loop transfer used for the control-loop design assumes that the boost converter is working in CCM. Under $50 \mathrm{~W}$, the boost is working in discontinuous conduction mode (DCM) and the frequency response of the converter changes.

In order to apply the $\{\mathrm{TR}\} 2$ regulation technique in the secondary side of the converter, an auxiliary transformer has been implemented in an RM14 core, using 3C90 material with four turns in the primary windings and 20 turns in the secondary windings ( $N R=5)$. Besides, two additional windings of four turns have been wrapped around the main core $(\mathrm{NM}=0.5)$.

The voltage control loop design has been designed and following the classical guidelines presented. The proposed converter has been first simulated by the power electronics simulation software (PSIM) and the results have been compared with experimental results. Figure show experimental and simulation results of the main switching waveforms on the resonant stage. It can be observed that the circuit is correctly tuned and each MOSFET works at $100 \mathrm{kHz}$ with a zero-current and zero-voltage commutation characteristics, with almost zerocommutation losses. 
Figure shows experimental results of the main-switching waveforms on the resonant stage for different input voltages. It can be observed that ZVZC switching is not affected under different input voltages (Vds of the main switches change is proportional to twice the input voltage). That is because the magnetizing current of the main transformer changes proportionally to the input voltage, needing the same gap time to charge and discharge the parasitic capacitances. As it can be seen in Figure, if the control voltage changes (bottom two waveforms), the resonance is not affected either (the two waveforms on the top). This is the main advantage of introducing the $\{\mathrm{TR}\} 2$ regulation in the secondary side of the main converter, the control voltage does not interfere in the ZVZC switching.

Figure shows the experimental frequency response VO /VC (continuous line) compared to the calculated one (dashed line).

In Figure, it is presented the experimental open-loop transfer function frequency response of the converter Aol (continuous line) compared with the calculated one (dashed line). An experimental crossover frequency of $2.9 \mathrm{kHz}$ and a phase margin of 54。 have been measured in the prototype. Figure show good agreement between the experimental and theoretical results, validating the small-signal model derived in this paper. Figure shows the experimental output response against a 2 A load change. The measured efficiency of the converter with and without the regulation, for different input voltages and output power, is represented in Figure. The added postregulator only decreases the overall efficiency of the ZVZCPS converter by approximately $1.5 \%$.

\section{CONCLUSION}

A new high-efficiency regulation method for a ZVZCPS converter is presented. The regulation method is based on the parallel power-processing technique, handling only a percentage of the power, and therefore, results in much higher efficiency than a normal preregulator would have. The novelty of the presented converter is that regulation is achieved without affecting the resonance of the ZVZCPS in any working conditions. Smalland large-signal models of the converter have been developed and a low-power prototype has been implemented to validate the proposed models.

The measured efficiency of the converter and the estimated one of a conventionally regulated SMART converter. The estimation has been done assuming that the serial converter used in the SMART has the same efficiency that the auxiliary converter developed in Section IV, and the resonant converter efficiency is the one shown in Figure as unregulated converter. As can be seen, the efficiency has been improved by over $4 \%$. However, the cost paid in is a little increase of the size and weight of the converter, since an additional transformer and inductor is required. This size penalty depends on the percentage of the total power that must handle the auxiliary converter. Further, this regulation method has a wide range of applicability to almost of converters or inverters that use a transformer to produce an isolated output.

\section{REFERENCES}

1). I. Barbi and R. Gules, "Isolated DC-DC converters with high-output voltage for TWTA telecommunications satellite applications,” IEEE Trans. Power Electron, vol. 18, no. 4, pp. 975-984, Jul. 2003.

2). E.-H. Kim and B.-H. Kwon, "High step-up resonant push-pull converter with high-efficiency," IET Power Electron., vol. 2, no. 1, pp. 79-89, Jan. 2009.

3). J.-M. Kwon, E-H. Kim, B.-H. Kwon and K.-H. Nam, "High-efficiency fuel cell power conditioning system with input current ripple reduction,” IEEE Trans. Ind. Electron., vol. 56, no. 3, pp. 826-834, Mar. 2009.

4). J.-M. Kwon and B.-H. Kwon, "High step-up active-clamp converter with Input-current doubler and output-voltage doubler for fuel cell power systems," IEEE Trans. Power Electron., vol. 24, no. 1, pp. 826-834, Jan. 2009.

5). J. F. Chen, R. Y. Chen, and T. J. Liang, "Study and implementation of a Single-stage current-fed boost PFC converter with ZCS for high voltage applications,” IEEE Trans. Power Electron., vol. 23, no. 1, pp. 379-386, Jan. 2008.

6). S. Jalbrzykowski and T. Citko, "Current-fed resonant full-bridge boost DC/AC/DC converter," IEEE Trans. Ind. Electron., vol. 55, no. 1, pp. 1198-1205, Mar. 2008.

7). R.-Y. Chen, T.-J. Liang, J.-F. Chen, R.-L. Lin, and K.-C. Tseng, "Study and implementation of a current-fed fullbridge boost DC-DC converter with zero-current switching for high-voltage application,” IEEE Trans. Ind. Appl., vol. 44, no. 4, pp. 1218-1226, Jul./Aug. 2008.

8). E. Abib and H. Farzanehfard, "Zero-voltage transition current-fed full-bridge PWM converter," IEEE Trans. Power Electron., vol. 24, no. 4, pp. 1041-1047, Apr. 2009.

9). H. Wang, Q. Sun, H. Shun Hung Chu, S. Tapuchi, and A. Ioinovici, "A ZCS current-fed full-bridge PWM converter with self-adaptable soft-switching snubber energy,” IEEE Trans. Power Electron., vol. 24, no. 8, pp. 1977-1991, Aug. 2009.

10). A. J. Mason, D. J. Tschirhart, and P. K. Jain, "New ZVS phase shift modulated full-bridge converter topologies with adaptive energy storage for SOFC application," IEEE Trans. Power Electron., vol. 23, no. 1, pp. 332-342, Jan. 2008 . 